\title{
Nuclear Fluorescence Using High-energy Synchrotron Radiation
}

\author{
D. Peter Siddons ${ }^{\mathrm{a}}$, Klaus-Dieter Liss ${ }^{\mathrm{b}}$, J. B. Hastings ${ }^{\mathrm{c}}$ and G. Grübel ${ }^{\mathrm{d}}$ \\ ${ }^{a}$ Brookhaven National Laboratory, Upton, New York 11973, USA. \\ ${ }^{b}$ Technische Universität Hamburg-Harburg,D21073, Hamburg, Germany. \\ ${ }^{c}$ Stanford Linear Accelerator, Stanford, CA94025 \\ ${ }^{d}$ HASYLAB, Deutches Elektronen-Synchrotron, Hamburg, Germany.
}

\begin{abstract}
We present some measurements of nuclear fluorescence which were made while exploring the potential of a unique source of very hard x-rays which was installed at ESRF until recently. It produced radiation of energies up to $1 \mathrm{MeV}$. In this range there are many nuclear resonances, and so we made a small survey of some plausible candidates to see if they could indeed be excited using this source. Along with short-lived resonances, we were also able to indirectly populate long-lived isomeric states in several nuclei. Thus, simply exposing the samples to the white beam for a period of time caused them to be activated. The samples could them be removed from the beam and their emissions observed using an energy-resolving detector. Isomeric states in isotopes of indium, cadmium and silver were excited and their subsequent decay observed.
\end{abstract}

\section{INTRODUCTION}

The first reference to nuclear fluorescence stimulated by monochromatic x-rays was a paper by Seppi and Bohm in $1962^{1}$. They used a high-power $\mathrm{x}$-ray generator in combination with a curved transmission monochromator designed by Dumond. They observed emission from ${ }^{19} \mathrm{~F}$ and ${ }^{55} \mathrm{Mn}$. This observation was completely overtaken by the excitement following the publication of Mossbauer's results on recoil-free gamma emission. We were impressed by their achievement, and wondered if such experiments could be done better using the high-brightness of SR. Some of us repeated those experiments at NSLS in 1989. More recently one of the present authors (DPS) had the opportunity to use an ESRF beamline producing much harder radiation than was available at NSLS, and was motivated to explore some of the higher energy levels which could only be attempted using that facility. In the midst of those experiments, a paper appeared in $\mathrm{PRL}^{2}$ describing efforts to excite an isomeric state in Hafnium. A sample of that isotope was not available to us. Examination of the Nuclear Data Tables revealed several possible other candidate isomeric states and so we explored those reactions also. This paper documents those attempts and presents our results.

\section{NUCLEAR FLUORESCENCE}

Nuclear fluorescence excited by synchrotron radiation offers the possibility of producing a clean, singly-excited system, in contrast to Bremsstrahlung-stimulated sources which indiscriminately excite all accessible levels. The high brightness of the SR source makes it possible to monochromate the radiation efficiently. This is not possible for a Bremsstrahlung source. All of the data presented here were collected at beamline ID15 using the superconducting wiggler source on the ESRF ring. We used a silicon (111) or (311) monochromator in a horizontal dispersion geometry. Although this is not ideal from a resolution point of view, we had insufficient time to build a vertically dispersing setup. Nevertheless we were able to observe nuclear emission form several isotopes. The scattered radiation was detected by an intrinsic Ge cryogenic detector set at a scattering angle of around 300 degrees, I.e. nearly backscattering. This was done in order to reduce as much as possible the elastic scattering by minimizing the

CP705, Synchrotron Radiation Instrumentation: Eighth International Conference, edited by T. Warwick et al.

(C) 2004 American Institute of Physics 0-7354-0180-2/04/\$22.00

1178 
atomic form factor. The Compton scattering is not suppressed $b$ this choice, but it is energy-shifted well away from the elastic line and so does not overwhelm the measurement of the fluorescence signal.
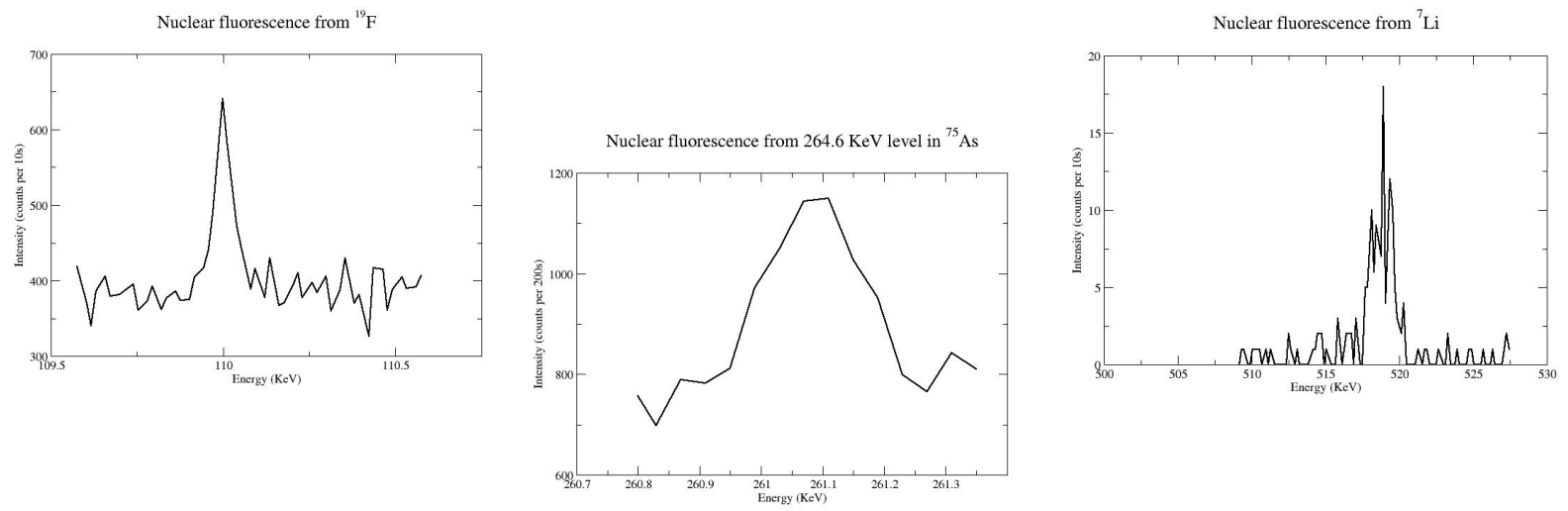

FIGURE 1. Energy scans through nuclear resonances in ${ }^{19} \mathrm{~F},{ }^{75} \mathrm{As}$ and ${ }^{7} \mathrm{Li}$. The $\mathrm{F}$ and As scans have a significant background which is absent in the Li scan. We believe this background comes from Compton scattering of higher-order beam components. The single-reflection monochromator used could not discriminate against such harmonics.

\section{LONG-LIVED ISOMERIC STATES}

Many isotopes have states for which the only possible decay path is highly forbidden. As a result these states are very long-lived, ranging from microseconds to years. We identified some plausible candidates which could be populated by an intermediate transition from a higher energy level, which in turn could be directly excited by SR. Most of the higher levels are fast, giving them a wide bandwidth and increasing the effective flux for pumping. We chose levels with lifetimes greater than a few seconds, ranging from 40 s to $5 \mathrm{~h}$. This allowed us to use an extremely simple approach. We placed the sample to receive the white beam directly or through a filter. It was exposed for some time, typically several half-lives, the beam turned off and the sample removed to a gamma counting station set up outside the hutch. Depending on the lifetime, spectra were acquired for a long time to record all the decay in one spectrum, or in short consecutive acquisitions to allow us to measure a decay curve.

\section{Silver}
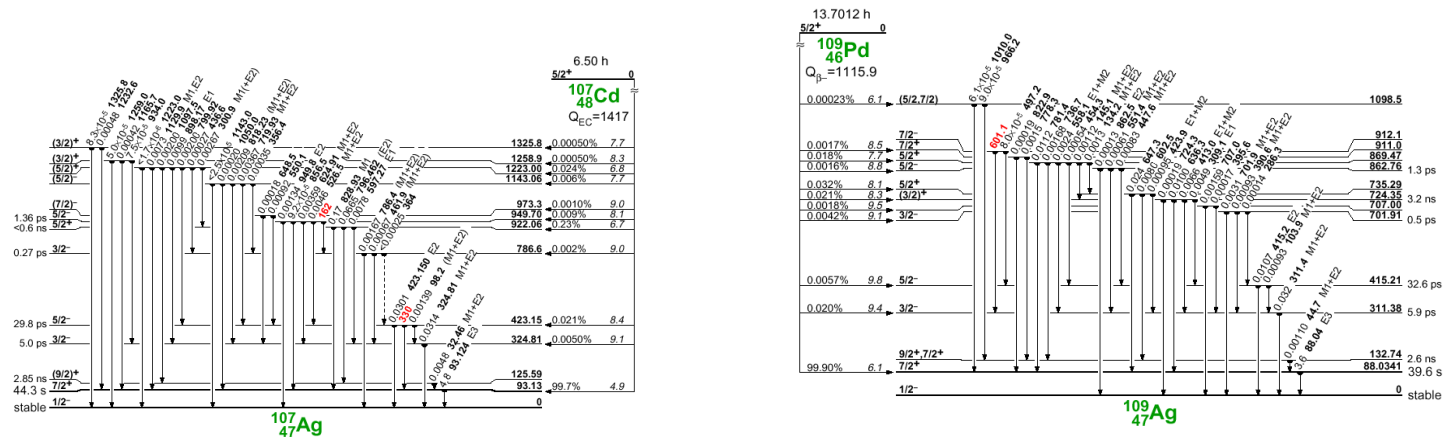

FIGURE 2. Decay schemes $^{3}$ for the two most abundant isotopes in silver. Each has an isomeric transition with a lifetime around 40 seconds. The long-lived states can be indirectly populated by exciting one of the short lifetime resonances of higher energy which has a decay branch to the level. 


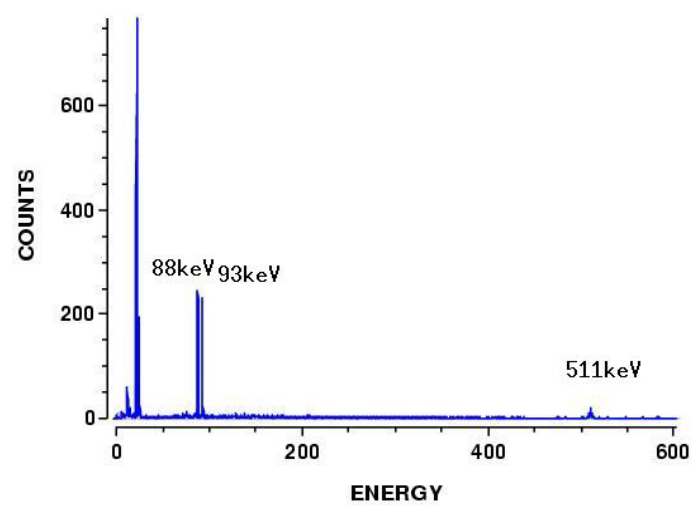

FIGURE 3. The emission spectrum from a silver foil which has been exposed to the high-energy white beam for several minutes. The low-energy lines around $22 \mathrm{keV}$ are $\mathrm{Ag} \mathrm{K}$ electronic fluorescence. The lines labeled 88 and $93 \mathrm{keV}$ are the lines due to the decay of the long-lived states in each of the two isotopes. The $511 \mathrm{keV}$ level appears to be annihilation radiation, and appears in all of the spectra we collected from these long-lived systems.
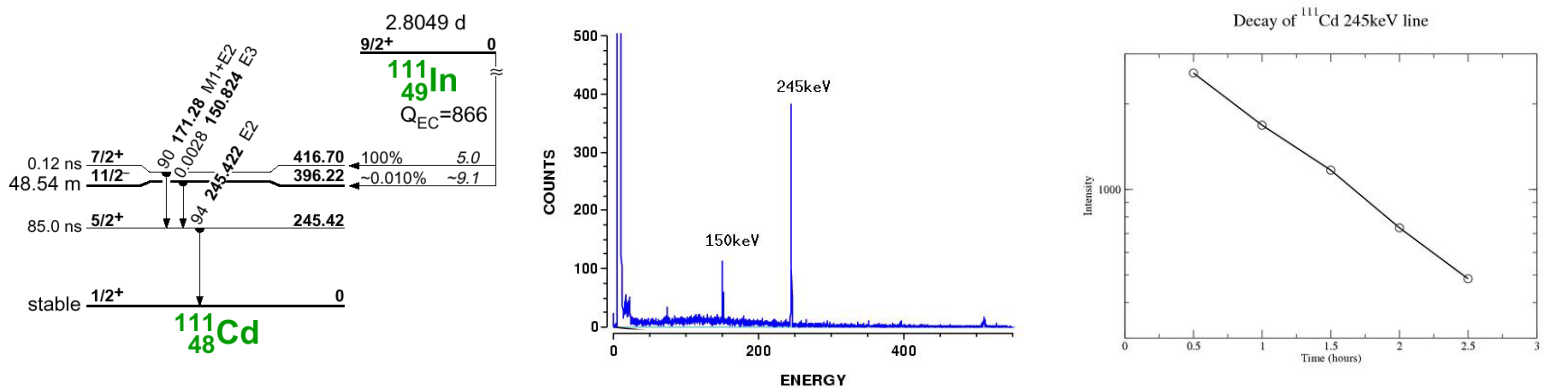

FIGURE 4. Decay scheme, emission spectrum and decay curve for ${ }^{111} \mathrm{Cd}$. The two labeled lines correspond to the cascade decay of the long-lived state through the $245 \mathrm{kV}$ level. This is similar to the process used in Cd-based PAC measurements, but in this case, no expensive isotope production is required.

\section{Indium}
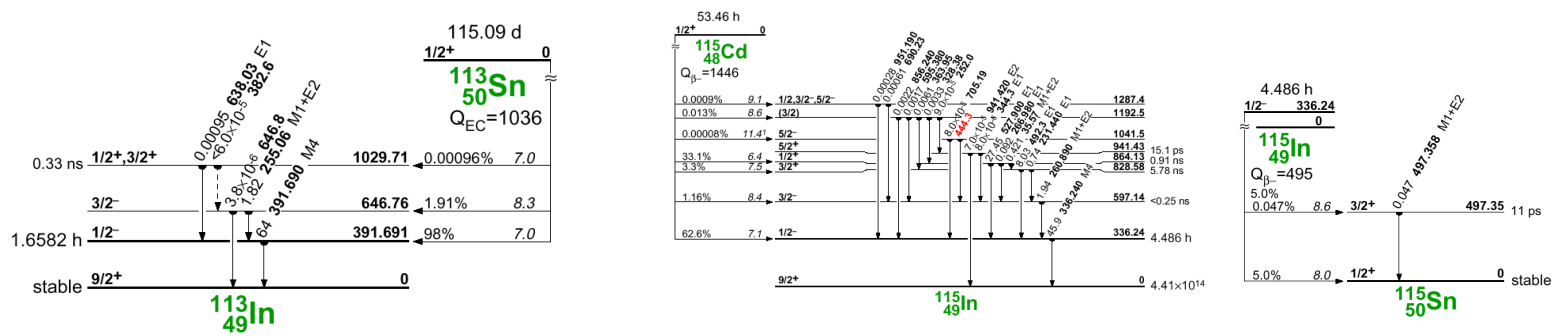

FIGURE 5. Decay schemes for the main isotopes of indium. ${ }^{113}$ In produces a line at $391 \mathrm{keV}$ with $1.6 \mathrm{hr}$ half-life. ${ }^{115} \mathrm{In}$ produces a line at $336 \mathrm{keV}$ with a $4.5 \mathrm{hr}$ half life. This excited state can also decay via beta-decay to produce an excited state of ${ }^{115} \mathrm{Sn}$. The $497 \mathrm{keV}$ line produced by this process is also observed. This is SR-induced transmutation. 


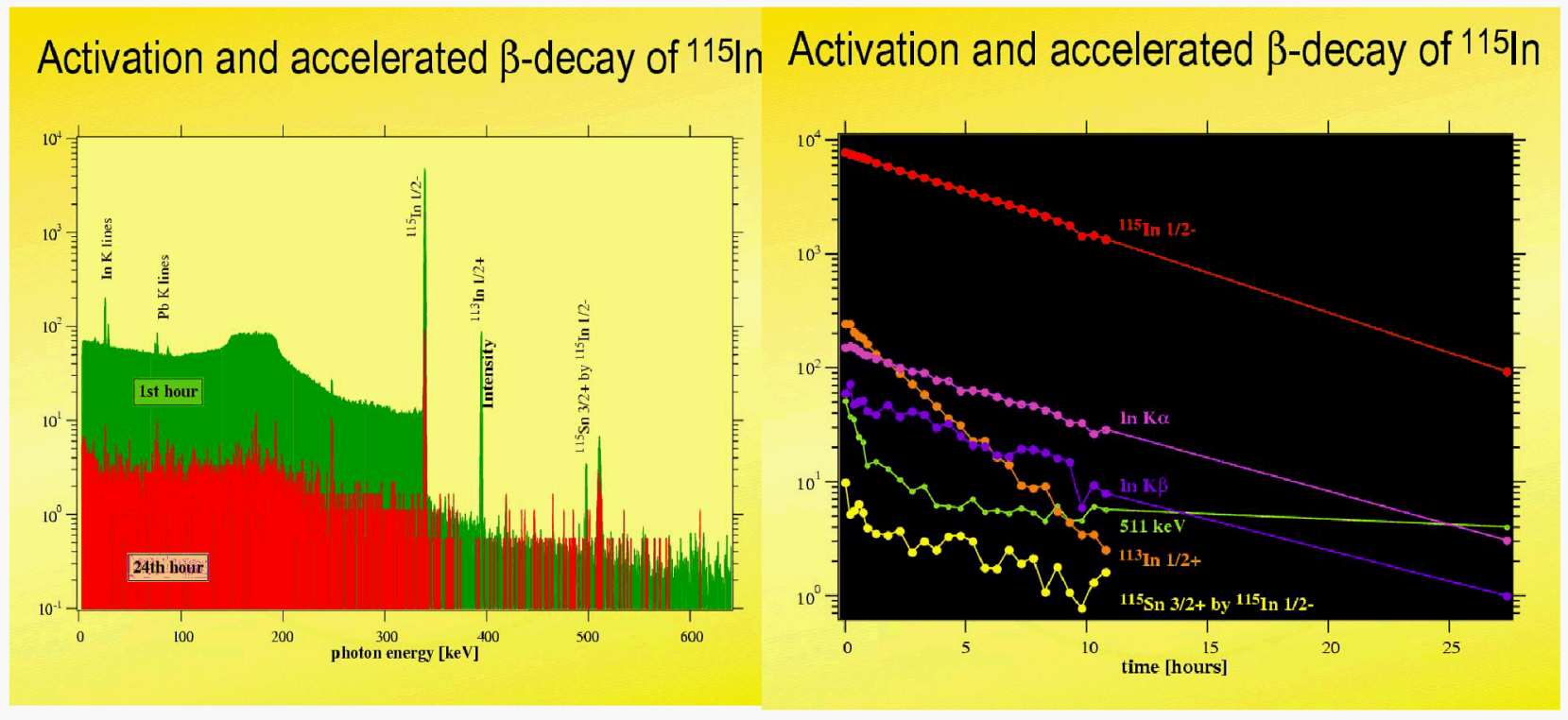

FIGURE 6. Observed spectra (left) and decay curves (right) for the various emission lines following irradiation of an indium foil sample.

We also attempted to study the $123 \mathrm{keV}$ state in ${ }^{176} \mathrm{Lu}$, one of the isotopes used for astronomical dating. Its isomeric state decays into ${ }^{176} \mathrm{Hf}$ with a $3.6 \mathrm{~h}$ lifetime, compared to the natural lifetime of $3.8 \times 10^{10}$ years. We could find no evidence for any enhanced decay following irradiation.

\section{ACKNOWLEDGMENTS}

One of us (DPS) is indebted to the ESRF for hosting him during a sabbatical leave (1998-1999) during which time this work was performed.

\section{REFERENCES}

\footnotetext{
1 E. J. Seppi \& F. Boehm, Phys. Rev. Let. 128 (1962) 2334

${ }^{2}$ C. B. Collins et al., Phys. Rev. Lett. 82 (1999) 695

${ }^{3}$ Decay scheme diagrams used by permission of John Wiley \& Sons, Inc. Taken from “Table of Isotopes, $8^{\text {th }}$ Edition (ISBN 0471-35633-6).
} 